\begin{tabular}{lc} 
Sharif University of Technology \\
Scientia Iranica \\
SCIENTIA & Transactions B: Mechanical Engineering \\
IRAN ICA & www.scientiairanica.com \\
\hline
\end{tabular}

\title{
Numerical simulation of the effect of visitor's movement on bacteria-carrying particles distribution in hospital isolation room
}

\author{
J. Eslami ${ }^{\mathrm{a}}$, A. Abbassi ${ }^{\mathrm{a}, *}$ and M.H. Saidi ${ }^{\mathrm{b}}$ \\ a. Department of Mechanical Engineering, Amirkabir University of Technology, 424 Hafez Ave., Tehran, P.O. Box 15916-34311, \\ Iran. \\ b. Center of Excellence in Energy Conversion (CEEC), School of Mechanical Engineering, Sharif University of Technology, Azadi \\ Ave, Tehran, P.O. Box 11155-9567, Iran.
}

Received 9 February 2016; received in revised form 2 May 2016; accepted 2 July 2016

\section{KEYWORDS \\ Isolation room; \\ Visitor walking; \\ Bacteria transport; \\ Dynamic mesh; \\ Ventilation \\ effectiveness.}

\begin{abstract}
The aim of this paper is to simulate numerically the airflow induced by a walking visitor and its effects on the contaminant transport and ventilation system effectiveness. To this end, the following models will be used in this study: the Lagrangian Discrete Random Walk (DRW) model to trace the motion of BCPs, the dynamic mesh method to simulate the visitor movement, and the Reynolds Averaged Navier-Stokes (RANS) model to solve the airflow. The validation results of the numerical method are in full agreement with the available experimental data in the literature. The findings of the present study indicate that the visitor's movement has remarkable effect on the basic airflow, and the increase of the visitor moving speed can decrease the risk of infection in the AIIR. It is also found that the concentration of BCPs in the back of visitor exceeds $10 \mathrm{cfu} / \mathrm{m}^{3}$, and the small distance between the patient and visitor has a negative impact on increasing the BCPs infection of the patient in AIIR. At the same time, it is observed that the effect of walking speed on the ventilation effectiveness index is not remarkable.
\end{abstract}

(C) 2017 Sharif University of Technology. All rights reserved.

\section{Introduction}

Some of the human health concerns necessitate strict observation and control over infection transmission within hospitals for infectious diseases, such as measles, chickenpox, Severe Acute Respiratory Syndrome (SARS), tuberculosis (TB), and influenza [1]. To implement appropriate infection control measures and prevent the transmission of contagious diseases from a patient to other people or from outsiders, such

*. Corresponding author. Tel.: +98 2164543425 ;

Fax: +982166419736

E-mail addresses: jabereslami@aut.ac.ir (J. Eslami); abbassi@aut.ac.ir (A. Abbassi); Saman@sharif.edu (M.H. Saidi) as health care personnel and visitors, to the susceptible patient, hospitals must have Airborne Infection Isolation Room (AIIR) which is a safe and healthy environment for patients with infectious diseases [2].

During patient care in the AIIR, the patient may be exposed to bacterial contamination. The main source of contamination in such ultra-clean environments can be categorized into two groups:

1. Exogenous sources such as hospital health care personnel, visitors, and the quality of the ventilation air;

2. Endogenous sources such as bacteria microscopic organisms on the patient's skin, mucous layers, or gastrointestinal tract [3].

Bacteria-Carrying Particles (BCPs) are considered as 
one of the major risk factors in spreading airborne diseases shed ding from health care workers and visitors, such as skin scales or squamae $[4,5]$.

It is clear that the contaminant distribution in AIIRs has a significant relationship with the main factors such as airflow pattern, particle characteristics, the location of obstacles [6], contaminant source location [7], supply/exhaust openings arrangement [8], and occupants and their motion. Investigations have shown that occupants and their motions are one of the most important parameters which may affect Indoor Air Quality (IAQ) as well as ventilation performance [9] and have an important influence on contaminant transport $[10,11]$ in ultra-clean environments.

Moving occupants in the AIIRs can disrupt the unidirectional downward airflow pattern and affect the BCPs transmission. Both experimental [12-16] and numerical methods [5,9,10,17-22] have been adopted to study the induced airflow pattern due to obstacle motion. Since experimental measurement is timeconsuming and very expensive, Computational Fluid Dynamics (CFD) techniques have become powerful and efficient tools for studying engineering problems, including airflow and contaminant distribution in enclosures [10].

Most of the previous studies have mainly concentrated on patient exhalation, coughing or sneezing; to the authors' best knowledge, few investigations have considered the moving object as a contamination source in the AIIR. Considering the implementation of the dynamic mesh technique, the aim of this research is to investigate numerically the airflow induced by a visitor's movement and its influence on the airflow pattern, contamination distribution and ventilation system performance within the downward unidirectional AIIR. To this end, this study tries to investigate the following issues:

1. The effect of visitor's movement on airflow pattern and BCPs distribution in the downward unidirectional AIIR model;

2. The role of different walking speeds on the BCPs concentration distribution around the patient body and the appropriate distance between visitor and patient to preserve the low infection level zone around the patient;

3. The functional relationship of the visitor movement on the ventilation effectiveness index.

Ultimately, the goal of this study is to make recommendations for a better design of the future isolation rooms.

\section{Model description}

To investigate the impact of a moving visitor on the airflow pattern, ventilation system performance, and

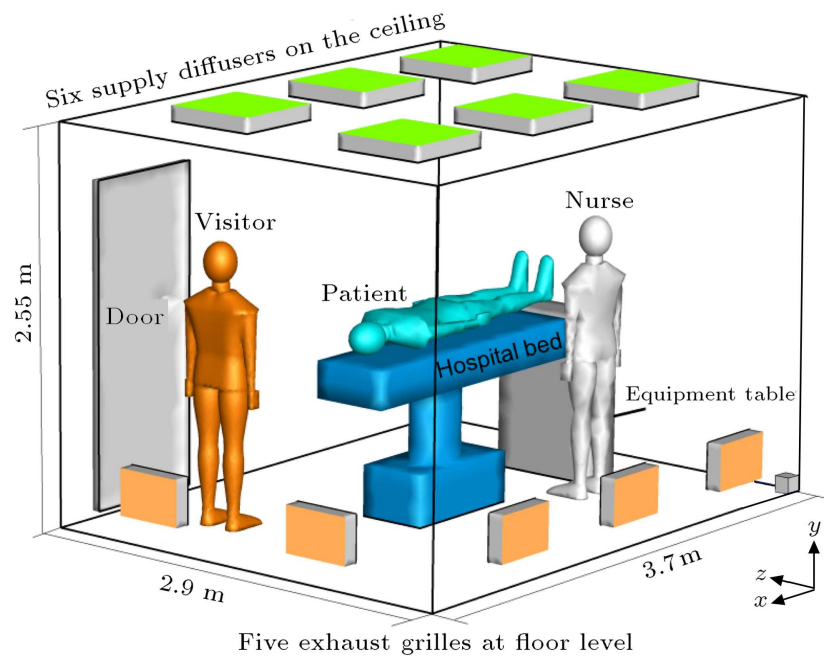

Figure 1. Schematic of the investigated AIIR model.

contaminant transport in an AIIR, a full-scale room is selected for simulation. Figure 1 displays the geometry of the physical AIIR model. As shown in Figure 1, the dimensions of the investigated AIIR model are $2.9 \times$ $3.7 \times 2.55 \mathrm{~m}^{3}$ (width $\times$ length $\times$ height). The model rooms are ventilated with six standard $0.6 \times 0.6 \mathrm{~m}^{2}$ (width $\times$ length) supply diffusers, located at the ceiling level. Five $0.45 \times 0.3 \mathrm{~m}^{2}$ (width $\times$ height) exhaust grills located on the cross vertical walls exhaust the airflow to return air channel.

There is one hospital bed whose dimensions are $0.5 \times 1.9 \times 1.05 \mathrm{~m}^{3}$ (width $\times$ length $\times$ height) in the middle of the AIIR. An equipment table is located next to the hospital bed. Its dimensions are $0.7 \times 0.3 \times$ $1.05 \mathrm{~m}^{3}$ (width $\times$ length $\times$ height). Three manikins with the dimensions of $0.5 \times 0.25 \times 1.8 \mathrm{~m}^{3}$ (width $\times$ length $\times$ height) are considered as a visitor, nurse, and a patient in the AIIR. The patient is laid on the hospital bed. It is assumed that the nurse and the patient are stationary all the time.

In the current research, it is also supposed that the visitor's body is the only source to spread out the contaminant particles in the AIIR. In this investigation, four steady walking speeds of $0.25,0.5,0.75$, and $1 \mathrm{~m} / \mathrm{s}$ are respectively performed to explore the influences of a moving visitor on the airflow field, ventilation performance, and contaminant concentration distribution. At first, the visitors are stationary in front of the entrance of the AIIR at Position $1(x=3.25 \mathrm{~m})$, and the ventilation air flows steadily. At time $t>0$, the visitor moves along the direction of $x$ from Position 1 towards Position $2(x=1.31 \mathrm{~m})$ with straight line motion, and then turns right and stops in Position 3 for two seconds opposite the nurse. On the way back, the visitor turns left (Position 4) and moves to get to the final station (Position 5) $(x=2.91 \mathrm{~m})$. According to the different walking speeds, the simulation times are different, but the total moving distance is $3.54 \mathrm{~m}$ (see Figure 2). 

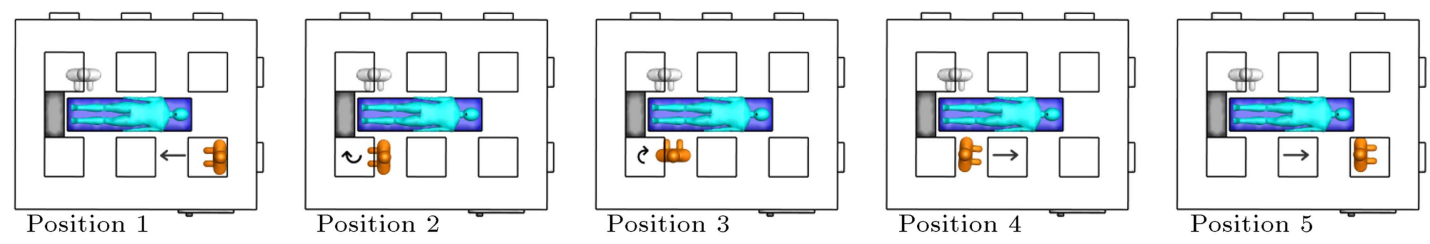

Figure 2. Schematic of different positions of the moving route.

\section{Mathematical model}

\subsection{Governing equations for airflow modeling}

Given that the particulate phase concentration in AIIRs is low, the airflow hydrodynamics is not influenced by the particulate phase. There is no interaction between continuous airflow phase and particle discrete phase; thus, the particles are treated as one-way coupling [23]. The governing equations of the air phase, including the conservation of mass, conservation of momentum, turbulent kinetic energy, $k$, and dissipation rate, $\varepsilon$, can be expressed in the general format as follows [24]:

$$
\frac{\partial}{\partial t}(\rho \varphi)+\operatorname{div}\left(\rho \vec{V} \varphi-\Gamma_{\varphi} \operatorname{grad} \varphi\right)=S_{\varphi}
$$

In this equation, $\rho$ is air density, $\vec{V}$ is the velocity vector, and $\varphi$ denotes the dependent variables (where $\varphi=1, u, v, w, k, \varepsilon)$. More details of effective diffusion coefficient, $\Gamma_{\varphi}$, and source term, $S_{\varphi}$, for each dependent variable are given by [25].

\subsection{Governing equations for particulate phase modeling}

In this simulation work, apart from the Eulerian approach for solving the airflow field, the Lagrangian DRW model is adopted to track the contaminant particles. The Lagrangian method is used to track the particles movement after being released from the surface of the visitor's body. To calculate trajectory of discrete phase particle in Lagrangian particle tracking approach, the equation of particle motion can be written as follows [24]:

$$
\frac{d u_{i}^{p}}{d t}=\frac{1}{\tau}\left(u_{i}^{a}-u_{i}^{p}\right)+\frac{g_{i}\left(\rho_{p}-\rho_{a}\right)}{\rho_{p}}+F_{a}
$$

where $u_{i}^{a}$ and $u_{i}^{p}$ are the air and the particles velocities, $\rho_{a}$ and $\rho_{p}$ are the density of air and the particles, $g_{i}$ and $F_{a}$ are the gravity and additional forces, respectively. The additional forces which may be included in the equation of motion are pressure gradient force, Saffman lift force caused by shear, virtual mass and Basset forces due to unsteady flow, thermophoretic and Brownian forces caused by temperature gradient [24]. In this research, based on the literature [24,26], the additional forces with the exception of drag force and gravitation, all forces are assumed negligible due to the relatively large size of the investigated particles. Particle relaxation time, $\tau$, is defined as [24]:

$$
\tau=\frac{S d_{p}^{2} C_{c}}{18 \nu},
$$

where $d_{p}$ is the diameter of the particle, $S$ is the ratio of particle density to fluid density, and $C_{c}$ is the Cunningham slip correction factor [24] implemented to compensate for non-continuum effects:

$$
C_{c}=1+\mathrm{Kn}\left[2.514+0.8 e^{\left(\frac{-1.1}{2 K \mathrm{Kn}}\right)}\right],
$$

where Knudsen number is defined as:

$$
\mathrm{Kn}=\frac{\lambda}{d_{p}} \text {. }
$$

In Eq. (5), $\lambda$ is the air mean free path which equals $68 \mathrm{~nm}$ in the normal conditions $\left(T=25^{\circ} \mathrm{C}\right.$ and $P=$ $1 \mathrm{~atm})$.

Since the turbulence diffusion is one of the most important features of the indoor airflow, its accurate estimation is necessary to predict the particle trajectory. In this study, Lagrangian DRW model [24] is adopted which simulates the components of the turbulent fluctuating velocity as follows:

$$
u_{i}^{\prime}=\xi \sqrt{{u^{\prime}}_{i}^{2}}=\xi \sqrt{\frac{2 k}{3}},
$$

where $\xi$ is a Gaussian random number, and $\sqrt{u^{\prime 2}}$ represents the Root Mean Square (RMS) of the fluctuating velocity components. For $k-\varepsilon$ family turbulence models, the RMS components are the same and equal to $2 / 3 \mathrm{k}$.

To compare the influence of walking speed on the ventilation effectiveness of the removal of BCPs, the Sandberg's mean ventilation efficiency index [27] is used which can be expressed in the following form:

$$
\eta=\frac{C_{o}-C_{i}}{C_{m}-C_{i}}
$$

where $C_{o}, C_{i}$, and $C_{m}$ are the concentration at the exhaust, supply, and room-averaged concentration of contaminant in a ventilated room, respectively. As the air supply system in an AIIRs provides clean air, say $C_{i}=0$, Eq. (7) reduces to:

$$
\eta=\frac{C_{o}}{C_{m}} \text {. }
$$




\subsection{Dynamic mesh technique}

To simulate the aerodynamic effects of the visitor's motion on the AIIR, the dynamic mesh model is employed for the numerical solution. The integral form of the corresponding governing equations for the dynamic mesh technique on control volume $V$ with boundary $\partial V$ is described as follows [24]:

$$
\begin{aligned}
\frac{d}{d t} \int_{V} \rho \varphi d V & +\int_{\partial V} \rho \varphi\left(\vec{V}-\vec{V}_{g}\right) \cdot d \vec{A} \\
& =\int_{\partial V} \Gamma_{\varphi} \nabla \varphi \cdot d \vec{A}+\int_{V} S_{\varphi} d V
\end{aligned}
$$

where $\vec{V}$ and $\overrightarrow{V_{g}}$ represent the airflow velocity vector and the mesh velocity of the moving mesh, respectively. More details can be found in [24]. In dynamic mesh zone, the remeshing method is adopted to remesh the agglomerated cells or faces, and smoothing method is used to adjust the mesh of a zone with a moving boundary [24].

\section{Numerical method}

The present study adopts the ANSYS Fluent commercial software to simulate the AIIR model. Lagrangian Discrete Random Walk (DRW) model is adopted to trace the motion of BCPs; similarly, the dynamic mesh technique is adopted to model the visitor's movement, the Reynolds Averaged Navier-Stokes (RANS) model employed to solve the airflow, and the Renormalization Group (RNG) $k-\varepsilon$ model adopted to model the turbulent flow inside the AIIR due to its accuracy and reputation for robustness [28].

\subsection{Solution procedure}

In the present study, ICEM-CFD meshing software (Ansys Inc.) is adapted to mesh the AIIR. The type of meshes is tetrahedral, and the mesh independency test is conducted using three different mesh densities (342 k (coarse), $613 \mathrm{k}$ (medium), and $1211 \mathrm{k}$ (fine)). In this study, a numerical uncertainty analysis is performed using the Grid Convergence Index (GCI) [29] to estimate the uncertainties resulting from mesh size.
For the chosen grid size, the GCI value for velocity magnitudes is around $4.12 \%$. Therefore, the medium grid density is fine enough to predict the airflow field and concentration distribution in the domain. The Finite Volume Method (FVM) is applied to discretize the conservation equations. A second-order upwind scheme is used to discretize the convection terms, and the SIMPLE scheme is selected to couple pressure with velocity [30]. The convergence criteria of the air phase properties are assumed to be $1 \times 10^{-4}$.

\subsection{Boundary conditions}

The supply uniform velocity is taken $0.21 \mathrm{~m} / \mathrm{s}$ with a turbulent intensity of $5 \%$. At the exhaust grills, the outflow boundary conditions are used. All boundary walls are supposed to be adiabatic, and the no-slip velocity boundary condition is applied to solid walls. For RNG $k-\varepsilon$ model mentioned above, the standard wall function is applied as boundary condition at the AIIR walls [31]. Considering the high air change rate and due to the short residence of the visitor moving in the AIIR, the occupants' bodies are supposed to be adiabatic.

In the current study, it is also assumed that the visitor body is the only source to spread out the contaminant particles in the model AIIR. According to Rui et al. [32], the uniform release rate of bacterial particles is set to 600 bacteria colony/min from the surface of the visitor's body. As explained in the introduction, the major source of bacterial emission in the AIIR is the particles with the diameter of $5-10 \mu \mathrm{m}$. Liu et al. [33] found that different diameters ranged from 5 to $10 \mu \mathrm{m}$ have minor differences among the results; therefore, the $5 \mu \mathrm{m}$ particles are used with a density of $1000 \mathrm{~kg} / \mathrm{m}^{3}$. In the present Lagrangian modeling for particles tracking, the trajectories of particles vanish as they impact the AIIR walls, hospital bed, equipment table, and occupants. The particles leave the AIIR model from exhaust grills. The boundary conditions for the CFD simulation are given in Table 1 .

\section{Validation of the CFD simulation}

According to the literature [4], RNG $k-\varepsilon$ model has been successfully performed to simulate indoor airflow

Table 1. Numerical boundary conditions for simulation.

\begin{tabular}{lccc}
\hline \multicolumn{1}{c}{ Name } & Boundary type & Velocity & Lagrangian model \\
\hline AIIR walls & Wall & No slip & Trap \\
Supply diffusers & Velocity Inlet & $0.21 \mathrm{~m} / \mathrm{s}$ & Escape \\
Exhaust grilles & Outflow & - & Escape \\
Hospital bed and equipment table & Wall & No slip & Trap \\
Visitor & Wall & $0.25-1 \mathrm{~m} / \mathrm{s}$ & - \\
Patient and nurse & Wall & No slip & Trap \\
\hline
\end{tabular}


field. Since RNG $k-\varepsilon$ turbulence model has been well validated to simulate velocity field in the isolation rooms, only the particle phase modeling is validated in this study. The normalized concentration in a threedimensional model room is investigated numerically and compared against experimental data derived from the study of Chen et al. [34].

Dimensions of the 3-D model for the numerical study are $0.4 \times 0.8 \times 0.4 \mathrm{~m}^{3}$ (width $\times$ length $\times$ height). The fresh air enters the room through the inlet $0.04 \times 0.04 \mathrm{~m}^{2}$ (width $\times$ height) which is located on the centerline of the room and symmetrically exhausted from the outlets $0.04 \times 0.04 \mathrm{~m}^{2}$ (width $\times$ height) in the faced side, as shown in Figure 3. The Air Changes' rate per hour $(\mathrm{ACH})$ used for the model room is $10 / \mathrm{h}$; according to this $\mathrm{ACH}$, the inlet flow velocity is $0.225 \mathrm{~m} / \mathrm{s}$.

The number of tracking tested particles in the Lagrangian DRW concentration model is 300000 with $10 \mu \mathrm{m}$ diameter and $1400 \mathrm{~kg} / \mathrm{m}^{3}$ density. As shown in Figure 4, the comparison of normalized concentration between the Lagrangian DRW concentration results and experimental data exhibits a fairly good agreement with a mean relative difference of $14 \%$ and a maximum relative difference of $42 \%$.

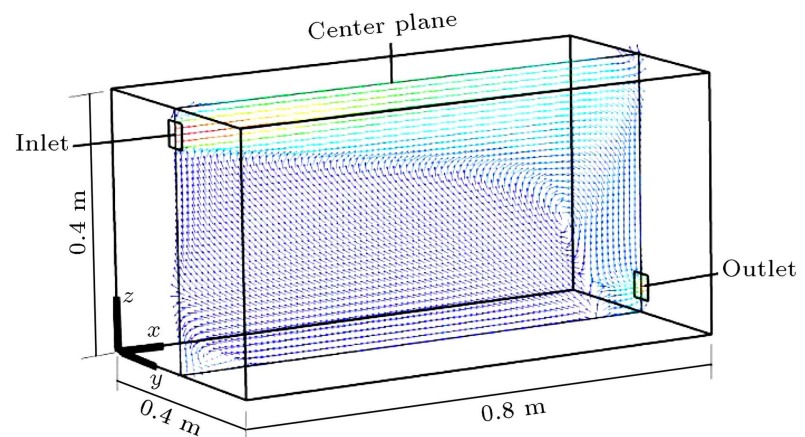

Figure 3. A schematic view of the experimental setup of Chen et al. [34].

\section{Result and discussion}

\subsection{Effect of visitor moving on airflow patterns}

Figure 5 shows the velocity vectors distribution of the AIIR induced by visitor movement at different walking speeds at plane $z=2.1 \mathrm{~m}$ when the visitor moves to Position 2. The following can be concluded based on Figure 5:

- The effect of the visitor movement on the basic airflow patterns is great and its motion pushes the indoor air in front of the visitor and creates a lowpressure zone in the back of the visitor. When the difference between visitor speed and supply diffuser velocity increases, the visitor's movement causes more disturbance in the main airflow pattern;

- The movement of the visitor can give rise to the formation of new recirculation zones: A larger zone behind the visitor and a smaller zone around the visitor's head which grows progressively as the visitor moves through the AIIR;

- When the moving speed increases from 0.25 to $1 \mathrm{~m} / \mathrm{s}$, the recirculation zone near the visitor's head becomes larger and demonstrates more changes in the downward unidirectional airflow.

\subsection{Effect of visitor moving on the concentration distribution of BCPs}

Recirculation zones due to the visitor's movement can entrap and entrain BCPs and create a high level of risk of infection in the AIIR. Figure 6 displays BCPs concentration distribution at plane $z=2.1 \mathrm{~m}$ for different walking speeds when the visitor moves to the final position (Position 5). The following can be drawn from Figure 6:

- The general features of the contaminant distribution for different walking speeds are almost similar to each other;

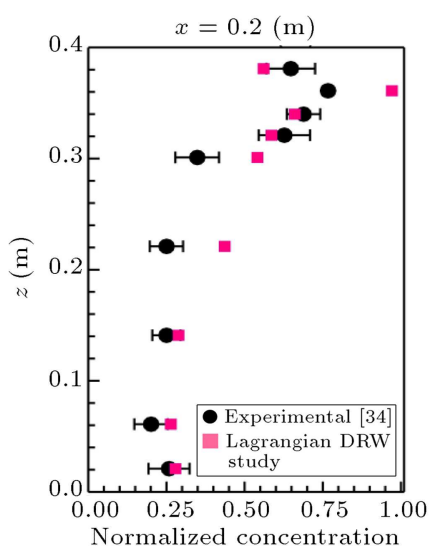

(a)

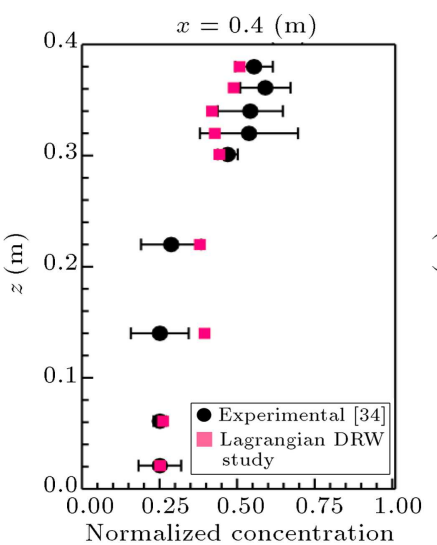

(b)

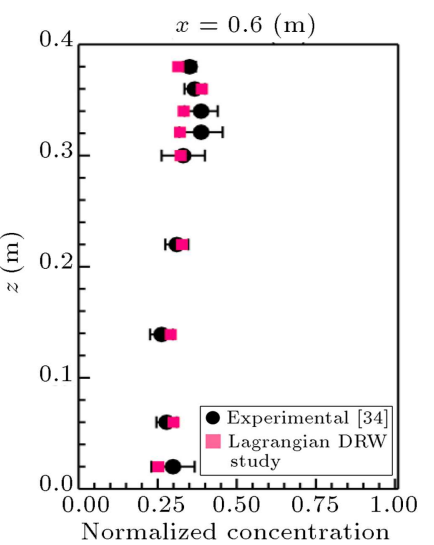

(c)

Figure 4. A comparison of experimental data [34] and simulated normalized concentrations by Lagrangian approach at three different locations. 


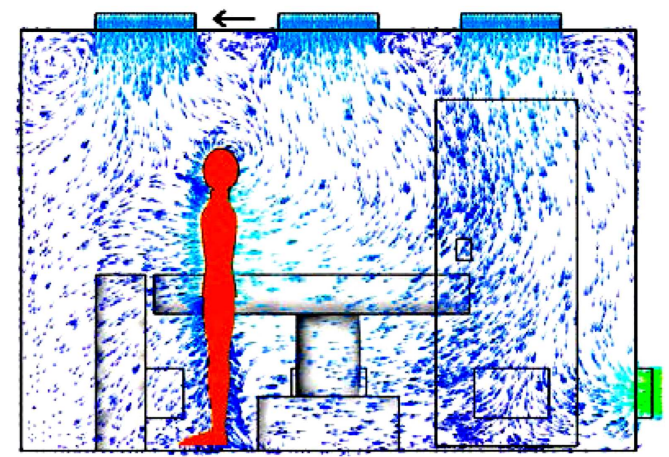

(a) $v=0.25 \mathrm{~m} / \mathrm{s}$, Position $2, t=7.75 \mathrm{~s}$

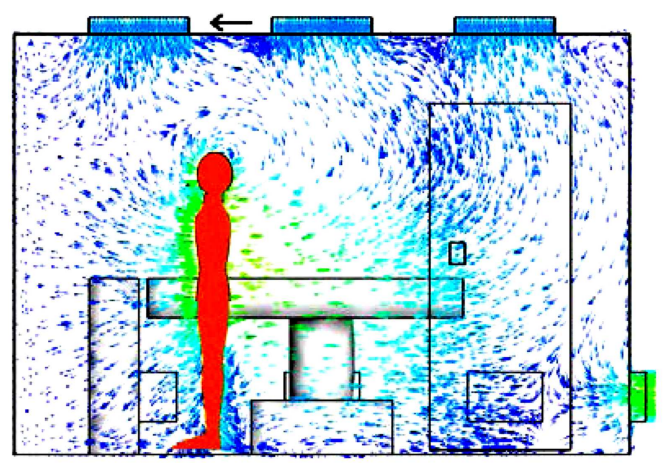

(c) $v=0.75 \mathrm{~m} / \mathrm{s}$, Position $2, t=2.58 \mathrm{~s}$

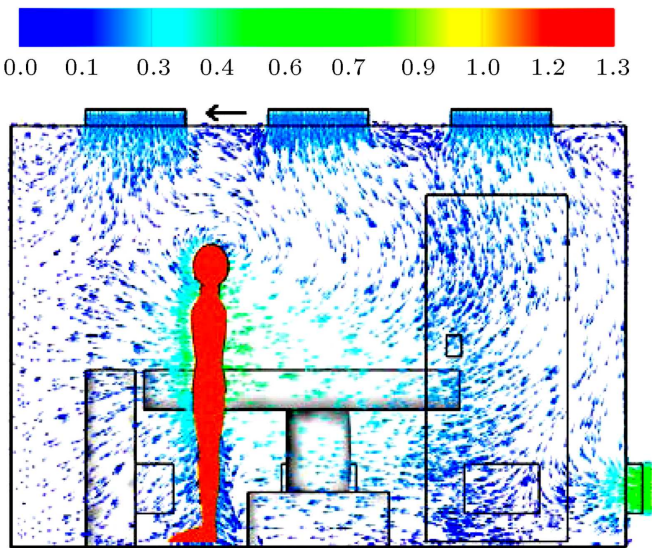

(b) $v=0.5 \mathrm{~m} / \mathrm{s}$, Position $2, t=3.88 \mathrm{~s}$

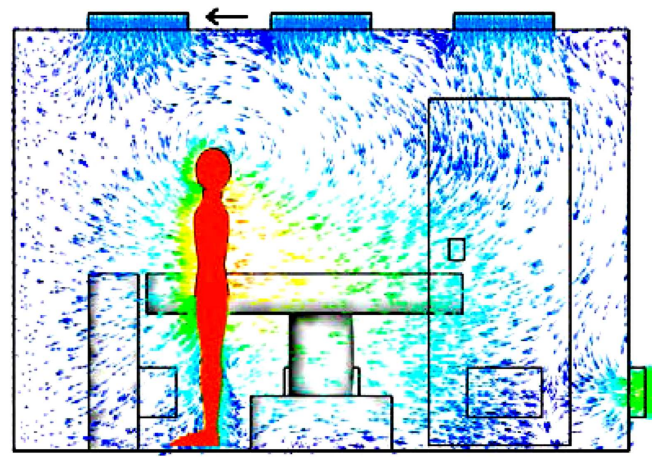

(d) $v=1 \mathrm{~m} / \mathrm{s}$, Position $2, t=1.94 \mathrm{~s}$

Figure 5. Velocity vectors distribution at $z=2.1 \mathrm{~m}$ for the different walking speeds of the visitor from Position 1 to 2.

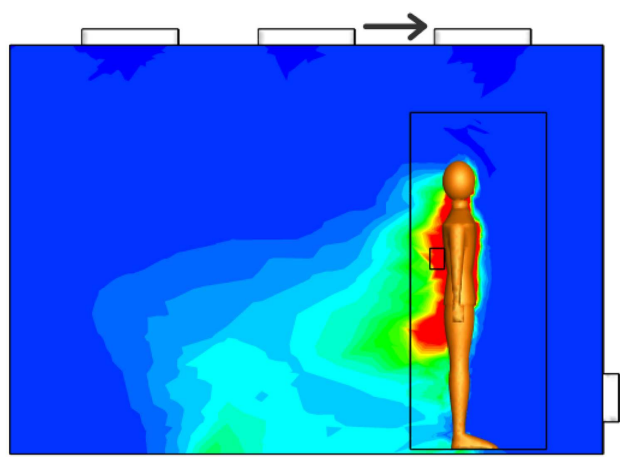

(a) $v=0.25 \mathrm{~m} / \mathrm{s}$, Position $5, t=18.15 \mathrm{~s}$

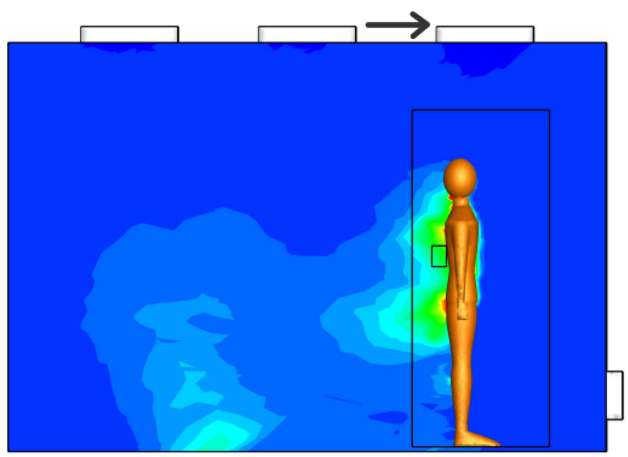

(c) $v=0.75 \mathrm{~m} / \mathrm{s}$, Position $5, t=8.72 \mathrm{~s}$

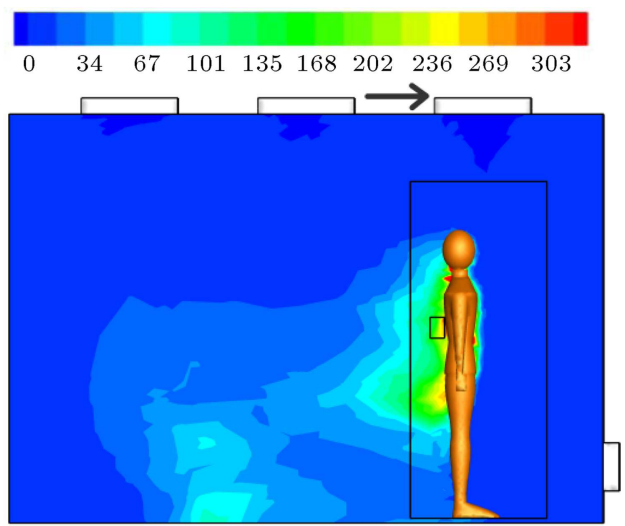

(b) $v=0.5 \mathrm{~m} / \mathrm{s}$, Position $5, t=11.1 \mathrm{~s}$

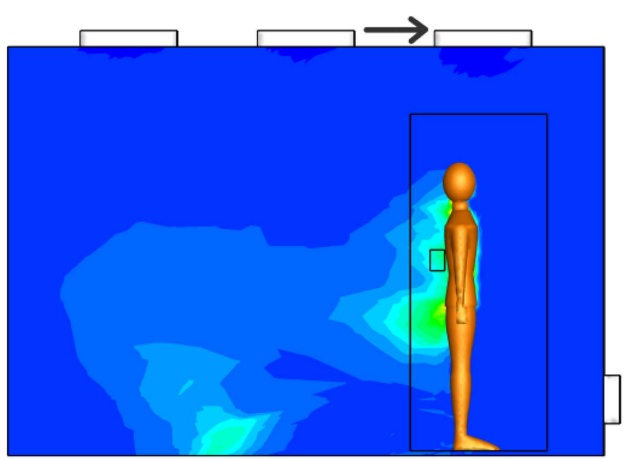

(d) $v=1 \mathrm{~m} / \mathrm{s}$, Position $5, t=7.54 \mathrm{~s}$

Figure 6. BCPs concentration $\left(\mathrm{cfu} / \mathrm{m}^{3}\right)$ at $z=2.1 \mathrm{~m}$ for different walking speeds when the visitor moves to Position 5 . 
- It can be seen that for all walking speeds, the concentration of BCPs at the back of the visitor becomes more than 10 colony forming units $\left(\mathrm{cfu} / \mathrm{m}^{3}\right)$;

- Due to the BCPs release from visitor body, it is obviously observed that for a slower walking speed, the zone near the back of the visitor becomes heavily contaminated and the infection risk increases.

For comprehensive investigation of the concentration of the BCPs near the patient, Figure 7 displays the transient distribution of BCPs at plane $y=1.05 \mathrm{~m}$ for visitor walking speed of $0.25 \mathrm{~m} / \mathrm{s}$ at different time instants. The following can be concluded based on Figure 7:

- It is observed that when the visitor walks forward, the contaminant distribution behind the visitor is rather local and the zone around the patient is not highly concentrated;

- It is found that on the way back, the visitor's movement and turning can cause the BCPs transport from the back of the visitor to the patient breathing zone and may increase the risk of patient's infection in this AIIR model.

Based on the above discussion, it can be said that

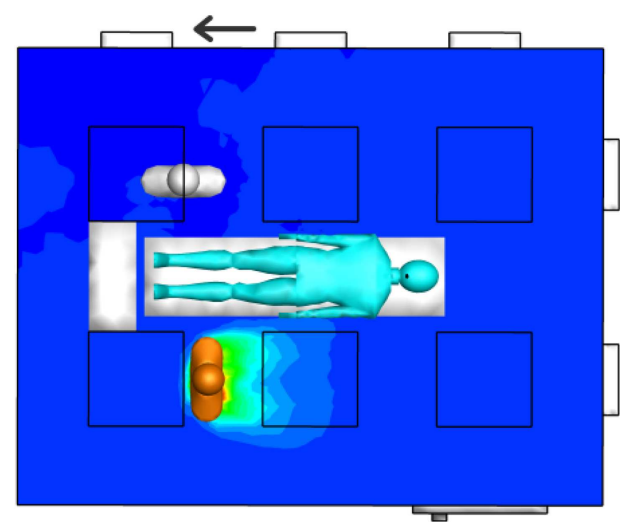

(a) $v=0.25 \mathrm{~m} / \mathrm{s}$, Position $2, t=7.75 \mathrm{~s}$

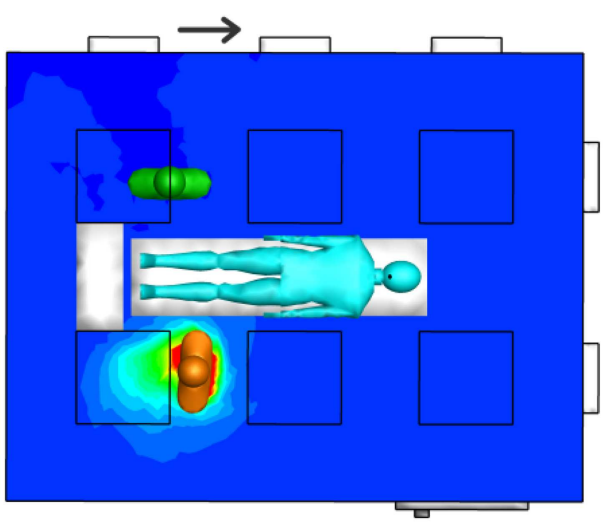

(c) $v=0.25 \mathrm{~m} / \mathrm{s}$, Position $4, t=11.75 \mathrm{~s}$ the visitor's movement has a remarkable effect on the deposition of BCPs. The BCPs may be deposited over critical surfaces, including the patient and nurse bodies, hospital bed, and instrument table by a mechanism, such as turbulent fluctuations. Figure 8 compares the deposited BCPs mass on the critical area for different

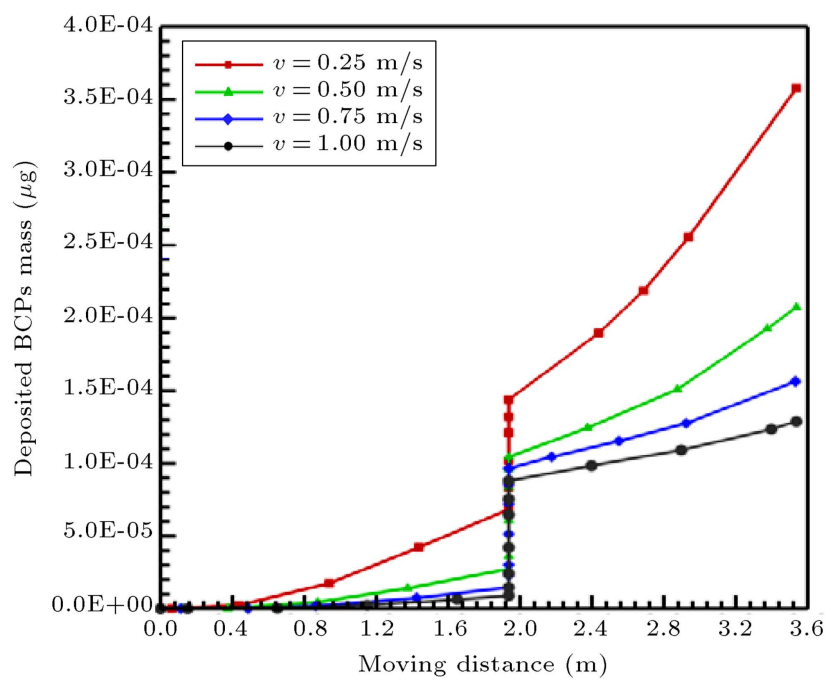

Figure 8. Comparison of the mass of deposited particles on critical area for different walking speeds.

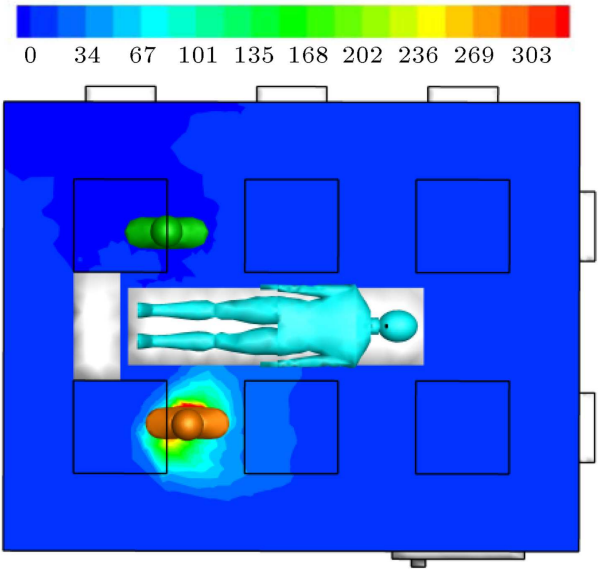

(b) $v=0.25 \mathrm{~m} / \mathrm{s}$, Position $3, t=8.75 \mathrm{~s}$

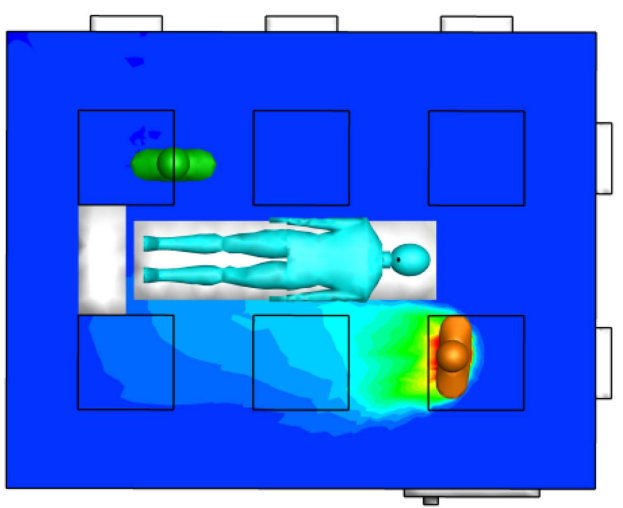

(d) $v=0.25 \mathrm{~m} / \mathrm{s}$, Position $5, t=18.15 \mathrm{~s}$

Figure 7. BCPs concentration $\left(\mathrm{cfu} / \mathrm{m}^{3}\right)$ at $y=1.05 \mathrm{~m}$ and visitor walking speed of $0.25 \mathrm{~m} / \mathrm{s}$ at different positions. 


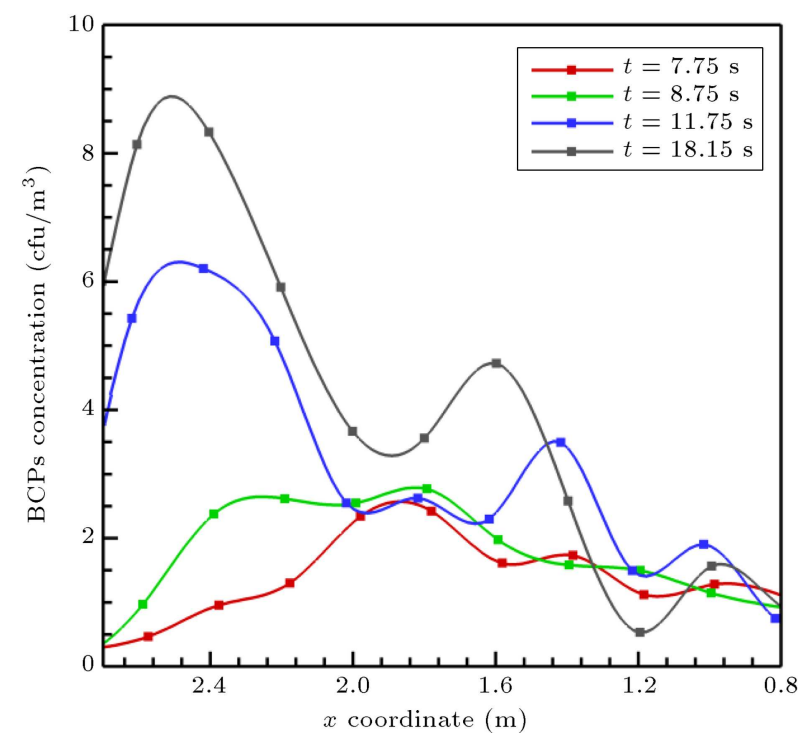

Figure 9. BCPs concentration for line interval along hospital bed $(x, y, z)=(0.8-2.7,1.38,1.45) \mathrm{m}$ and walking speed of $v=0.25 \mathrm{~m} / \mathrm{s}$ at different time instants.

walking speeds. In addition, to investigate the infection risk near the patient, Figure 9 shows the local BCPs concentration along the patient when the walking speed is $0.25 \mathrm{~m} / \mathrm{s}$. The line interval is located at the height of the patient face which is at $y=1.38 \mathrm{~m}$. The following highlights the trends in Figures 8 and 9:

- The faster walking speed cases have lower mass of deposited BCPs on the critical area. In fact, there is little contamination on the patient for faster walking speed which implies less risk of infection (see Figure 8);

- In this AIIR model during visitor's movement, the BCPs concentration at the patient's breathing zone remains less than $10 \mathrm{cfu} / \mathrm{m}^{3}$ due to the appropriate distance between the patient and visitor and downward unidirectional airflow (see Figure 9).

To make a prediction of critical BCPs infection area around the visitor, Figure 10 shows the comparison of BCPs concentration between different walking speeds when the visitor moves to Position 5. The following highlights the trends in Figure 10:

- It can be seen that the distance between the patient and visitor is very important to prevent the BCPs infection, and the small distances between them can increase the infection risk of the patient. For instance, when the distance between the patient and visitor decreases from $440 \mathrm{~mm}$ to $200 \mathrm{~mm}$, the BCPs concentration can increase as much as $1000 \%$;

- It is found that when the distance between the patient and visitor is more than $440 \mathrm{~mm}$, the visitor's movement does not have a highly significant influence on the patient. On the other hand, in the

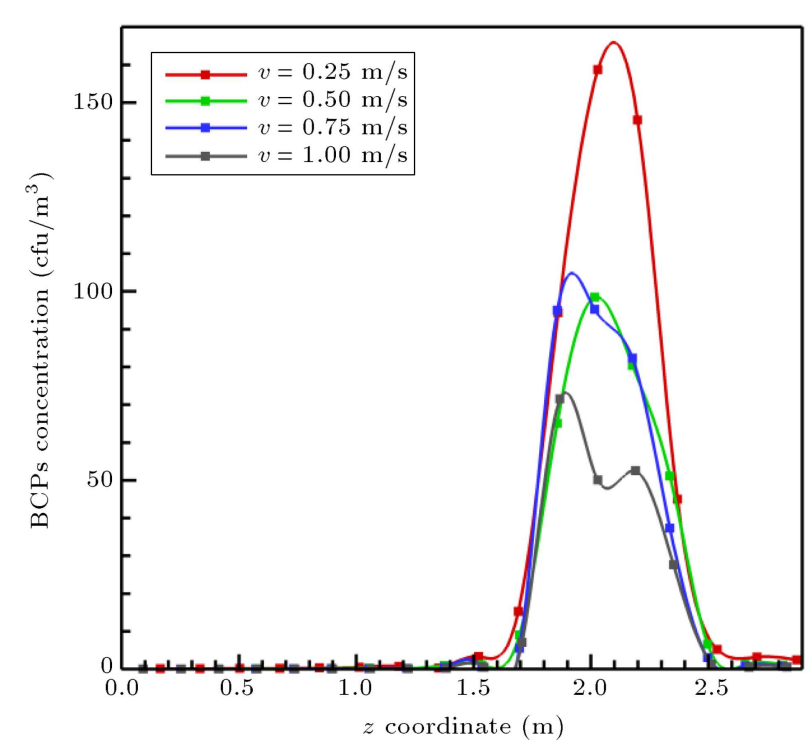

Figure 10. BCPs concentration profiles along a horizontal line along hospital bed $(x, y, z)=(2.5,1.38,0-2.9) \mathrm{m}$ for different moving speeds.

critical distances within $440 \mathrm{~mm}$ around the visitor, the air with more than $10 \mathrm{cfu} / \mathrm{m}^{3}$ can be observed which increases the risk of infection.

\subsection{Effect of visitor moving on the ventilation system effectiveness}

Figure 11 represents a quantitative comparison of the impact of different visitor walking speeds on the ventilation effectiveness of the system. This can be defined as a percentage of released particles evacuated by the ventilation airflow. Based on the different visitor walking speeds, the results are presented as a function of the visitor position in Figure 11(a) and the instant time in Figure 11(b). According to Figure 11, we may realize that:

- For different walking speeds, ventilation effectiveness gradually increases as the visitor moves across the room and magnifies when the visitor moves slower, especially at moving speed of $0.25 \mathrm{~m} / \mathrm{s}$. Nevertheless, the influence of visitor walking speeds on the ventilation system performance is not significant and the maximum value reaches $1.63 \%$.

\section{Conclusions}

In this paper, the effect of a visitor walking on the concentration of airborne BCPs shedding from visitor in an AIIR was examined numerically using unsteady CFD simulations. Also, its influence on the airflow pattern and ventilation effectiveness was investigated. Four different walking speeds of $0.25,0.5,0.75$, and $1 \mathrm{~m} / \mathrm{s}$ were considered in this research. The conclusions are presented as follows:

1. The visitor's movement creates new recirculation 


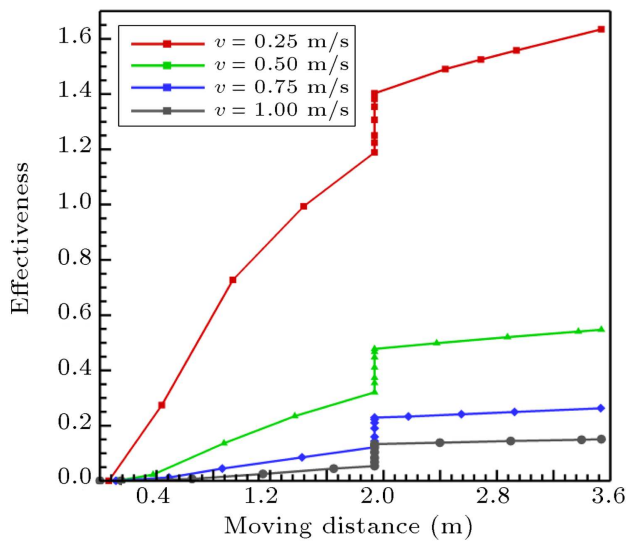

(a)

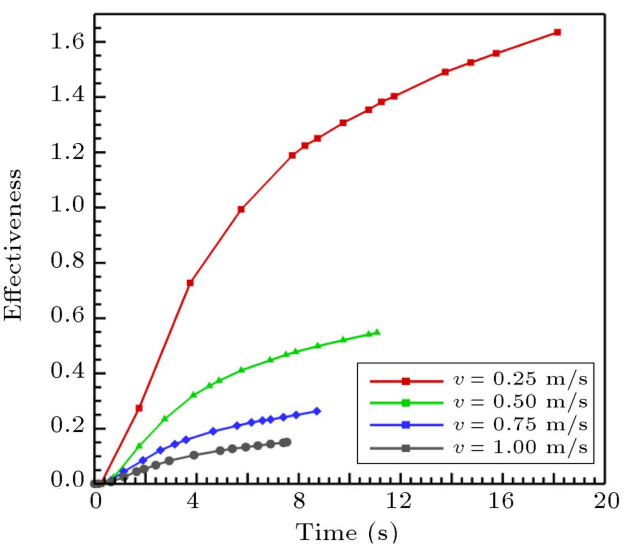

(b)

Figure 11. The effect of visitor's movement on the effectiveness of ventilation system versus (a) moving distance and (b) time.

zones which may entrain and entrap the airborne BCPs released from the visitor's body in the AIIR and change the infection level around the patient. For smaller walking speeds, the back of the visitor becomes heavily contaminated and the infection risk increases;

2. The distance between the patient and visitor is very important to preserve the low infection level zone around the patient. For this AIIR model, it is suggested that the minimum distance between them must be $440 \mathrm{~mm}$ to decrease the infection risk of the patient. On the other hand, when the distance between the patient and visitor is more than $440 \mathrm{~mm}$, the visitor's movement does not have a remarkable influence on the air flow near the patient;

3. The effect of walking speed on the effectiveness of ventilation system is not remarkable and maximum value reaches $1.63 \%$.

The present study and its results lead to superior comprehension of the impact of moving visitor on the airflow pattern and contaminants dispersion in AIIR and may find useful applications to design more effective ventilation strategies.

\section{Nomenclature}

\section{Abbreviations}

AIIR Airborne Infection Isolation Room

BCP Bacteria-Carrying Particle

CFD Computational Fluid Dynamics

CFU Colony Forming Units

DRW Discrete Random Walk

E-L Eulerian-Lagrangian

GCI Grid Convergence Index
IAQ Indoor Air Quality

RANS Reynolds Averaged Navier-Stokes

RMS Root Mean Square

RNG Renormalization Group

\section{Greek symbols}

Energy dissipation rate

$\rho \quad$ Density of the fluid

$\rho_{p} \quad$ Density of the particle

$\tau \quad$ Particle relaxation time

$\lambda \quad$ Mean free path

$\Gamma_{\varphi} \quad$ Effective diffusion coefficient

$\xi \quad$ Gaussian random number

$\varphi \quad$ Dependent variable

$\partial V \quad$ Boundary of the control volume

$\eta \quad$ Mean ventilation efficiency index

\section{Latin symbols}

$\begin{array}{ll}C_{o} & \text { Outlet concentration } \\ C_{i} & \text { Inlet concentration } \\ C_{m} & \text { Volume-averaged concentration } \\ C_{c} & \text { Cunningham slip correction factor } \\ d_{p} & \text { Particle diameter } \\ F_{a} & \text { Additional forces } \\ g_{i} & \text { Gravitational constant in } i \text { direction } \\ k & \text { Turbulent kinetic energy } \\ \text { Kn } & \text { Knudsen number } \\ P & \text { Pressure } \\ S & \text { Particle to fluid density ratio } \\ S_{\varphi} & \text { Source term } \\ T & \text { Temperature } \\ t & \text { Time } \\ u, v, w & \text { Velocity components }\end{array}$


$u_{i}$

$u_{i}^{p}$

$u_{i}^{\prime}$

$\vec{V}$

$\overrightarrow{V_{g}}$

V

$v$

$x, y, z$

\section{Subscripts}

$i, j \quad$ Spatial coordinates

$p \quad$ Particle

\section{References}

1. Hang, J., Li, Y., Ching, W.H., Wei, J., Jin, R., Liu, L. and Xie, X. "Potential airborne transmission between two isolation cubicles through a shared anteroom", Building and Environment, 89, pp. 264-278 (2015).

2. Qian, H. and Li, Y. "Removal of exhaled particles by ventilation and deposition in a multibed airborne infection isolation room", Indoor Air, 20(4), pp. 28497 (2010).

3. Reichman, D.E. and Greenberg, J.A. "Reducing surgical site infections: a review", Reviews in Obstetrics and Gynecology, 2(4), pp. 212-221 (2009).

4. Sadrizadeh, S., Tammelin, A., Ekolind, P. and Holmberg, S. "Influence of staff number and internal constellation on surgical site infection in an operating room", Particuology, 13, pp. 42-51 (2014).

5. Wang, J. and Chow, T.T. "Numerical investigation of influence of human walking on dispersion and deposition of expiratory droplets in airborne infection isolation room", Building and Environment, 46(10), pp. 1993-2002 (2011).

6. Lee, H. and Awbi, H.B. "Effect of internal partitioning on indoor air quality of rooms with mixing ventilationbasic study", Building and Environment, 39(2), pp. 127-141 (2004).

7. Zhong, K., Yang, X. and Kang, Y. "Effects of ventilation strategies and source locations on indoor particle deposition", Building and Environment, 45(3), pp. 655-662 (2010).

8. Méndez, C., San José, J.F., Villafruela, J.M. and Castro, F. "Optimization of a hospital room by means of CFD for more efficient ventilation", Energy and Buildings, 40(5), pp. 849-854 (2008).

9. Wu, Y. and Gao, N. "The dynamics of the body motion induced wake flow and its effects on the contaminant dispersion", Building and Environment, 82, pp. 63-74 (2014).

10. Mazumdar, S. and Chen, Q.Y. "Impact of moving bodies on airflow and contaminant transport inside aircraft cabins", in Proceedings of Roomvent (2007).

11. Matsumoto, H. and Ohba, Y. "The influence of a moving object on air distribution in displacement ventilated rooms", Journal of Asian Architecture and Building Engineering, 3(1), pp. 71-75 (2004).

12. Bjorn, E. and Nielsen, P.V. "Dispersal of exhaled air and personal exposure in displacement ventilated rooms", Indoor Air, 12(3), pp. 147-164 (2002).

13. Brohus, H., Balling, K.D. and Jeppesen, D. "Influence of movements on contaminant transport in an operating room", Indoor Air, 16(5), pp. 356-372 (2006).

14. Poussou, S.B., Mazumdar, S., Plesniak, M.W., Sojka, P.E. and Chen, Q. "Flow and contaminant transport in an airliner cabin induced by a moving body: Model experiments and CFD predictions", Atmospheric Environment, 44(24), pp. 2830-2839 (2010).

15. Brohus, H., Hyldig, M., Kamper, S. and Vachek, U. "Influence of persons' movements on ventilation effectiveness", in Proceedings of Indoor Air (2008).

16. Mattsson, M., Bjørn, E., Sandberg, M. and Nielsen, P.V., Simulating People Moving in Displacement Ventilated Rooms, Dept. of Building Technology and Structural Engineering (1997).

17. Shih, Y.C., Chiu, C.C. and Wang, O. "Dynamic airflow simulation within an isolation room", Building and Environment, 42(9), pp. 3194-3209 (2007).

18. Mazumdar, S., Yin, Y., Guity, A., Marmion, P., Gulick, B. and Chen, Q. "Impact of moving objects on contaminant concentration distributions in an inpatient ward with displacement ventilation", $H V A C \& R$ Research, 16(5), pp. 545-563 (2010).

19. Edge, B.A., Paterson, E.G. and Settles, G.S. "Computational study of the wake and contaminant transport of a walking human", Journal of Fluids Engineering, 127(5), pp. 967-977 (2005).

20. Hang, J., Li, Y. and Jin, R. "The influence of human walking on the flow and airborne transmission in a sixbed isolation room: Tracer gas simulation", Building and Environment, 77, pp. 119-134 (2014).

21. Deng, T., Zhang, Q. and Zhang, G. "The influences of moving human in a ventilation room", Building Simulation, pp. 779-786 (2007).

22. Wang, J. and Chow, T.T. "Influence of human movement on the transport of airborne infectious particles in hospital", Journal of Building Performance Simulation, 8(4), pp. 205-215 (2015).

23. Zhang, Z. and Chen, Q. "Comparison of the Eulerian and Lagrangian methods for predicting particle transport in enclosed spaces", Atmospheric Environment, 41(25), pp. 5236-5248 (2007).

24. Ansys., Fluent User Manual, Ansys Inc (2012).

25. Saidi, M.H., Sajadi, B. and Molaeimanesh, G.R. "The effect of source motion on contaminant distribution in 
the cleanrooms", Energy and Buildings, 43(4), pp. 966970 (2011).

26. Zhao, B., Zhang, Y., Li, X., Yang, X. and Huang, D. "Comparison of indoor aerosol particle concentration and deposition in different ventilated rooms by numerical method", Building and Environment, 39(1), pp. 1-8 (2004).

27. Sandberg, M. "What is ventilation efficiency?", Building and Environment, 16(2), pp. 123-135 (1981).

28. Zhang, Z., Chen, X., Mazumdar, S., Zhang, T. and Chen, Q. "Experimental and numerical investigation of airflow and contaminant transport in an airliner cabin mockup", Building and Environment, 44(1), pp. 85-94 (2009).

29. Roache, P.J. "Perspective: a method for uniform reporting of grid refinement studies", Journal of Fluids Engineering, 116(3), pp. 405-413 (1994).

30. Patankar, S., Numerical Heat Transfer and Fluid Flow, CRC Press (1984).

31. Launder, B.E. and Spalding, D.B. "The numerical computation of turbulent flows", Computer Methods in Applied Mechanics and Engineering, 3(2), pp. 269289 (1974).

32. Rui, Z., Guangbei, T. and Jihong, L. "Study on biological contaminant control strategies under different ventilation models in hospital operating room", Building and Environment, 43(5), pp. 793-803 (2008).

33. Liu, J., Wang, H. and Wen, W. "Numerical simulation on a horizontal airflow for airborne particles control in hospital operating room", Building and Environment, 44(11), pp. 2284-2289 (2009).

34. Chen, F., Yu, S.C.M. and Lai, A.C.K. "Modeling particle distribution and deposition in indoor environments with a new drift-flux model", Atmospheric Environment, 40(2), pp. 357-367 (2006).

\section{Biographies}

Jaber Eslami is a $\mathrm{PhD}$ candidate of Mechanical Engineering at Amirkabir University of Technology, Tehran, Iran. His research areas include modeling and numerical analysis of transport of aerosol particles, CFD simulations, and experimental measurements of a built environment.

Abbas Abbassi is a Professor of Mechanical Engineering at Amirkabir University of Technology, Tehran, Iran. His current research interests include convective heat and fluid flow, HVAC systems analysis and design, indoor air quality and clean room technology, and industrial energy audit.

Mohammad Hassan Saidi is a Professor in the School of Mechanical Engineering at Sharif University of Technology, Tehran, Iran. His current research interests include MEMS, heat transfer enhancement in boiling and condensation, modeling of pulse tube refrigeration, vortex tube refrigerators, indoor air quality and clean room technology, energy efficiency in home appliances, and desiccant cooling systems. 